Research Article

\title{
Solvability for Discrete Fractional Boundary Value Problems with a $p$-Laplacian Operator
}

\author{
Weidong Lv \\ School of Mathematics and Statistics, Longdong University, Qingyang, Gansu 745000, China \\ Correspondence should be addressed to Weidong Lv; lvweidong2004@163.com
}

Received 5 July 2013; Accepted 3 September 2013

Academic Editor: Jehad Alzabut

Copyright (C) 2013 Weidong Lv. This is an open access article distributed under the Creative Commons Attribution License, which permits unrestricted use, distribution, and reproduction in any medium, provided the original work is properly cited.

This paper is concerned with the solvability for a discrete fractional $p$-Laplacian boundary value problem. Some existence and uniqueness results are obtained by means of the Banach contraction mapping principle. Additionally, two representative examples are presented to illustrate the effectiveness of the main results.

\section{Introduction}

For any number $a \in \mathbb{R}$ and each interval $\mathbb{a}$ of $\mathbb{R}$, we denote $\mathbb{N}_{a}=\{a, a+1, a+2, \ldots\}$ and $\mathbb{\mathbb { N }}_{a}=\mathbb{\square} \cap \mathbb{N}_{a}$ throughout this paper. It is also worth noting that, in what follows, we appeal to the convention that the empty sum is taken to be 0 .

In this paper, we will consider the existence and uniqueness of solutions for the following discrete fractional boundary value problem involving a $p$-Laplacian operator

$$
\begin{gathered}
\Delta\left[\phi_{p}\left(\Delta_{C}^{\alpha} u\right)\right](t)=f(t+\alpha-1, u(t+\alpha-1)), \quad t \in[0, b]_{\mathbb{N}_{0}}, \\
u(\alpha-2)=\beta_{1} u(\alpha+b+1), \\
\Delta u(\alpha-2)=\Delta u(\alpha-1)=\beta_{2} \Delta u(\alpha+b),
\end{gathered}
$$

where $1<\alpha \leq 2, b \in \mathbb{N}_{1}, \beta_{1} \neq 1, \beta_{2} \neq 1, \Delta$ is the forward difference operator with stepsize $1, \Delta_{C}^{\alpha}$ denotes the discrete Caputo fractional difference of order $\alpha, f$ : $[\alpha-1, \alpha+b-1]_{\mathbb{N}_{\alpha-1}} \times \mathbb{R} \rightarrow \mathbb{R}$ is a continuous function, and $\phi_{p}$ is the $p$-Laplacian operator; that is, $\phi_{p}(u)=|u|^{p-2} u, p>1$. Obviously, $\phi_{p}$ is invertible and its inverse operator is $\phi_{q}$, where $q>1$ is a constant such that $1 / p+1 / q=1$.

The theory of fractional differential equations has become a new important branch of mathematics (see, e.g., [1-8]). At the same time, boundary value problems for fractional differential equations have received considerable attention [9-18]. It is well known that discrete analogues of differential equations can be very useful in applications $[19,20]$, especially for using computer to simulate the behavior of solutions for certain dynamic equations. Compared to continuous case, significantly less is known about the discrete fractional calculus. However, within the recent years, a lot of papers have appeared on discrete fractional calculus and discrete fractional boundary value problems; see [21-37]. For example, in [25], Atıc1 and Eloe explored a discrete fractional conjugate boundary value problem with the Riemann-Liouville fractional difference. To the best of our knowledge, this is pioneering work on discussing boundary value problems in discrete fractional calculus. After that, Goodrich studied discrete fractional boundary value problems involving the Riemann-Liouville fractional difference intensively and obtained a series of excellent results; see [26-31]. In [33, 34], Bastos et al. considered the discrete fractional calculus of variations and established several necessary optimality conditions for fractional difference variational problems. Abdeljawad introduced the conception of Caputo fractional difference and developed some useful properties of it in [35]. Ferreira in [37] initially investigated the existence and uniqueness of solutions for some discrete fractional boundary value problems of order less than one by the Banach fixed point theorem.

Very recently, some authors have focused their attention on the existence of solutions for fractional boundary value problems with the $p$-Laplacian operator in continuous case [38-44]. However, as far as we know, few papers can be found 
in the literature for the discrete fractional boundary value problems with the $p$-Laplacian operator [45].

Inspired by the aforementioned results, we will investigate the discrete fractional $p$-Laplacian boundary value problem (1) and establish some sufficient conditions for the existence and uniqueness of solutions to it by using the Banach contraction mapping principle.

The remainder of this paper is organized as follows. Section 2 preliminarily provides some necessary basic knowledge for the theory of discrete fractional calculus. In Section 3, the existence and uniqueness results for the solution to problem (1) will be established with the help of the contraction mapping principle. Finally, in Section 4, two concrete examples are provided to illustrate the possible applications of the established analytical results.

\section{Preliminaries}

For the convenience of the reader, we begin by presenting here some necessary basic definitions and lemmas on discrete fractional calculus theory.

Definition 1 (see [21]). For any $t$ and $v$, the falling factorial function is defined as

$$
t^{\underline{v}}=\frac{\Gamma(t+1)}{\Gamma(t+1-v)}
$$

provided that the right-hand side is well defined. We appeal to the convention that if $t+1-v$ is a pole of the Gamma function and $t+1$ is not a pole, then $t^{\underline{\nu}}=0$.

Definition 2 (see [46]). The $\nu$ th fractional sum of a function $f: \mathbb{N}_{a} \rightarrow \mathbb{R}$, for $v>0$, is defined by

$$
\Delta^{-v} f(t)=\frac{1}{\Gamma(\nu)} \sum_{s=a}^{t-v}(t-s-1)^{\frac{\nu-1}{}} f(s), \quad \text { for } t \in \mathbb{N}_{a+v} .
$$

Definition 3 (see [35]). The $v$ th Caputo fractional difference of a function $f: \mathbb{N}_{a} \rightarrow \mathbb{R}$, for $v>0, v \notin \mathbb{N}$, is defined by

$$
\begin{aligned}
\Delta_{C}^{v} f(t) & =\Delta^{-(n-v)} \Delta^{n} f(t) \\
& =\frac{1}{\Gamma(n-v)} \sum_{s=a}^{t-n+v}(t-s-1)^{\frac{n-\nu-1}{}} \Delta^{n} f(s), \\
& \text { for } t \in \mathbb{N}_{a+n-v},
\end{aligned}
$$

where $n$ is the smallest integer greater than or equal to $v$ and $\Delta^{n}$ is the $n$th forward difference operator. If $\nu=n \in \mathbb{N}$, then $\Delta_{C}^{v} f(t)=\Delta^{n} f(t)$.

Lemma 4 (see [45]). Assume that $v>0$ and $f$ is defined on $\mathbb{N}_{a}$. Then

$$
\Delta^{-v} \Delta_{C}^{v} f(t)=f(t)+c_{0}+c_{1} t+\cdots+c_{n-1} t^{\frac{n-1}{2}},
$$

where $c_{i} \in \mathbb{R}, i=1,2, \ldots, n-1$, and $n$ is the smallest integer greater than or equal to $v$.
Now, we state and prove the following lemma, which provides a representation for the solution to (1) if the solution exists.

Lemma 5. Let $h:[\alpha-1, \alpha+b-1]_{\mathbb{N}_{\alpha-1}} \rightarrow \mathbb{R}$, and let $\beta_{1}, \beta_{2} \neq 1$. Then the following problem

$$
\begin{gathered}
\Delta\left[\phi_{p}\left(\Delta_{C}^{\alpha} u\right)\right](t)=h(t+\alpha-1), \quad t \in[0, b]_{\mathbb{N}_{0}}, \\
u(\alpha-2)=\beta_{1} u(\alpha+b+1), \\
\Delta u(\alpha-2)=\Delta u(\alpha-1)=\beta_{2} \Delta u(\alpha+b),
\end{gathered}
$$

has a unique solution

$$
\begin{aligned}
& u(t)= \frac{a(t)}{\Gamma(\alpha-1)} \sum_{s=0}^{b+1}(\alpha+b-s-1)^{\frac{\alpha-2}{}} \phi_{q}\left(\sum_{\tau=0}^{s-1} h(\tau+\alpha-1)\right) \\
&+\frac{\beta_{1}}{\left(1-\beta_{1}\right) \Gamma(\alpha)} \\
& \times \sum_{s=0}^{b+1}(\alpha+b-s)^{\frac{\alpha-1}{}} \phi_{q}\left(\sum_{\tau=0}^{s-1} h(\tau+\alpha-1)\right) \\
&+\frac{1}{\Gamma(\alpha)} \sum_{s=0}^{t-\alpha}(t-s-1)^{\frac{\alpha-1}{}} \phi_{q}\left(\sum_{\tau=0}^{s-1} h(\tau+\alpha-1)\right), \\
& t \in[\alpha-2, \alpha+b+1]_{\mathbb{N}_{\alpha-2}},
\end{aligned}
$$

where $a(t)=\left(\beta_{2}\left\{\left[\beta_{1}(\alpha+b+1)+2-\alpha\right]+\left(1-\beta_{1}\right) t\right\}\right) /((1-$ $\left.\left.\beta_{1}\right)\left(1-\beta_{2}\right)\right)$.

Proof. The definition of the discrete Caputo fractional difference, together with condition $\Delta u(\alpha-2)=\Delta u(\alpha-1)$, implies that $\Delta_{C}^{\alpha} u(0)=0$. So from (6), we have

$$
\begin{aligned}
\phi_{p}\left(\Delta_{C}^{\alpha} u(t)\right) & =\phi_{p}\left(\Delta_{C}^{\alpha} u(0)\right)+\sum_{s=0}^{t-1} h(s+\alpha-1) \\
& =\sum_{s=0}^{t-1} h(s+\alpha-1),
\end{aligned}
$$

and then

$$
\Delta_{C}^{\alpha} u(t)=\phi_{q}\left(\sum_{s=0}^{t-1} h(s+\alpha-1)\right), \quad t \in[0, b+1]_{\mathbb{N}_{0}} .
$$

Hence, in view of Lemma 4, we can get

$$
\begin{aligned}
u(t)= & \frac{1}{\Gamma(\alpha)} \sum_{s=0}^{t-\alpha}(t-s-1)^{\frac{\alpha-1}{}} \phi_{q}\left(\sum_{\tau=0}^{s-1} h(\tau+\alpha-1)\right) \\
& +c_{0}+c_{1} t
\end{aligned}
$$

where $t \in[\alpha-2, \alpha+b+1]_{\mathbb{N}_{\alpha-2}}, c_{0}, c_{1} \in \mathbb{R}$. 
Furthermore, we have

$$
\begin{aligned}
\Delta u(t)= & \frac{1}{\Gamma(\alpha-1)} \sum_{s=0}^{t-(\alpha-1)}(t-s-1)^{\frac{\alpha-2}{} \phi_{q}} \\
& \times\left(\sum_{\tau=0}^{s-1} h(\tau+\alpha-1)\right)+c_{1}, \quad t \in[\alpha-2, \alpha+b]_{\mathbb{N}_{\alpha-2}} .
\end{aligned}
$$

Then by conditions $u(\alpha-2)=\beta_{1} u(\alpha+b+1), \Delta u(\alpha-2)=$ $\beta_{2} \Delta u(\alpha+b)$, we can get

$$
\begin{aligned}
c_{0}= & \frac{\beta_{1}}{\left(1-\beta_{1}\right) \Gamma(\alpha)} \\
& \times \sum_{s=0}^{b+1}(\alpha+b-s)^{\frac{\alpha-1}{}} \phi_{q}\left(\sum_{\tau=0}^{s-1} h(\tau+\alpha-1)\right) \\
& +\frac{\beta_{2}\left[\beta_{1}(\alpha+b+1)+2-\alpha\right]}{\left(1-\beta_{1}\right)\left(1-\beta_{2}\right) \Gamma(\alpha-1)} \\
& \times \sum_{s=0}^{b+1}(\alpha+b-s-1)^{\frac{\alpha-2}{2}} \phi_{q}\left(\sum_{\tau=0}^{s-1} h(\tau+\alpha-1)\right), \\
c_{1}= & \frac{\beta_{2}}{\left(1-\beta_{2}\right) \Gamma(\alpha-1)} \\
& \times \sum_{s=0}^{b+1}(\alpha+b-s-1)^{\frac{\alpha-2}{}} \phi_{q}\left(\sum_{\tau=0}^{s-1} h(\tau+\alpha-1)\right) .
\end{aligned}
$$

Substituting the values of $c_{0}$ and $c_{1}$ in (11), we get (8). This completes the proof.

Finally, we list below the following basic properties of the $p$-Laplacian operator which will be used in the sequel.

(1) If $1<p<2, u v>0$ and $|u|,|v| \geq m>0$, then

$$
\left|\phi_{p}(v)-\phi_{p}(u)\right| \leq(p-1) m^{p-2}|v-u| .
$$

(2) If $p>2,|u|,|v| \leq M$, then

$$
\left|\phi_{p}(v)-\phi_{p}(u)\right| \leq(p-1) M^{p-2}|v-u| .
$$

\section{Main Results}

In this section, we will use the Banach contraction mapping principle to prove the existence and uniqueness for the solution to problem (1).

Let $\mathbb{E}$ denote the Banach space of all functions from $[\alpha-$ $2, \alpha+b+1]_{\mathbb{N}}$ into $\mathbb{R}$ endowed with the norm defined by $\|u\|=\max \left\{|u(t)|, t \in[\alpha-2, \alpha+b+1]_{\mathbb{N}_{\alpha-2}}\right\}$. we set

For the sake of convenience to the following discussion,

$$
\bar{a}=\max \left\{|a(t)|, t \in[\alpha-2, \alpha+b+1]_{\mathbb{N}_{\alpha-2}}\right\},
$$

where $a(t)$ is as given in Lemma 5 . Also, for any $u, v \in \mathbb{E}$, we denote

$$
\begin{aligned}
A(v, u)(t)= & \phi_{q}\left(\sum_{s=0}^{t-1} f(s+\alpha-1, v(s+\alpha-1))\right) \\
& -\phi_{q}\left(\sum_{s=0}^{t-1} f(s+\alpha-1, u(s+\alpha-1))\right),
\end{aligned}
$$

for $t \in[0, b+1]_{\mathbb{N}_{0}}$. Obviously, $A(v, u)(0)=0$.

In view of Lemma 5 , we transform problem (1) as

$$
u=\mathscr{F} u \text {, }
$$

where $\mathscr{F}: \mathbb{E} \rightarrow \mathbb{E}$ is defined by

$$
\begin{aligned}
(\mathscr{F} u)(t)= & \frac{a(t)}{\Gamma(\alpha-1)} \sum_{s=0}^{b+1}(\alpha+b-s-1)^{\frac{\alpha-2}{}} \\
& \times \phi_{q}\left(\sum_{\tau=0}^{s-1} f(\tau+\alpha-1, u(\tau+\alpha-1))\right) \\
& +\frac{\beta_{1}}{\left(1-\beta_{1}\right) \Gamma(\alpha)} \sum_{s=0}^{b+1}(\alpha+b-s)^{\frac{\alpha-1}{2}} \\
& \times \phi_{q}\left(\sum_{\tau=0}^{s-1} f(\tau+\alpha-1, u(\tau+\alpha-1))\right) \\
& +\frac{1}{\Gamma(\alpha)} \sum_{s=0}^{t-\alpha}(t-s-1)^{\frac{\alpha-1}{2}} \\
& \times \phi_{q}\left(\sum_{\tau=0}^{s-1} f(\tau+\alpha-1, u(\tau+\alpha-1))\right)
\end{aligned}
$$

for $t \in[\alpha-2, \alpha+b+1]_{\mathbb{N}_{\alpha-2}}$. It is clear to see that $u$ is a solution of the problem (1) if and only if $u$ is a fixed point of $\mathscr{F}$.

Now, we state the main results as follows.

Theorem 6. Suppose $p>2, \beta_{1} \neq 1, \beta_{2} \neq 1$, and the following condition holds:

$\left(\mathrm{H}_{1}\right)$ there exist positive numbers $\lambda$ and $k$ with

$$
\begin{aligned}
k<\left(\left|1-\beta_{1}\right| b !\right) \times( & (q-1)(b+1) \\
& \times\left[\left|1-\beta_{1}\right|(\bar{a} \alpha+\alpha+b)+\left|\beta_{1}\right|(\alpha+b)\right] \\
& \left.\times[\lambda \Gamma(\alpha+1)]^{q-2} \prod_{i=1}^{b-1}(\alpha+i)\right)^{-1},
\end{aligned}
$$


such that

$$
\begin{aligned}
& \lambda \alpha t^{\frac{\alpha-1}{}} \\
& \quad \leq f(t, u), \quad \text { for }(t, u) \in[\alpha-1, \alpha+b-1]_{\mathbb{N}_{\alpha-1}} \times \mathbb{R}, \\
& |f(t, v)-f(t, u)| \\
& \quad \leq k|v-u|, \quad \text { for } t \in[\alpha-1, \alpha+b-1]_{\mathbb{N}_{\alpha-1}}, u, v \in \mathbb{R} .
\end{aligned}
$$

Then the problem (1) has a unique solution.

Proof. For any $u \in \mathbb{E}$, by (21), we can get that

$$
\begin{aligned}
\sum_{s=0}^{t-1} f(s+\alpha-1, u(s+\alpha-1)) \\
\quad \geq \lambda \sum_{s=0}^{t-1} \alpha(s+\alpha-1)^{\underline{\alpha-1}} \\
=\lambda(t+\alpha-1)^{\underline{\alpha}} \geq \lambda \Gamma(\alpha+1), \quad t \in[1, b+1]_{\mathbb{N}_{0}} .
\end{aligned}
$$

Due to $p>2$ and $1 / p+1 / q=1$, we know that $1<q<2$. By (14) and (22), for any $u, v \in \mathbb{E}, t \in[1, b+1]_{\mathbb{N}_{1}}$, we have

$$
\begin{aligned}
|A(v, u)(t)| \leq & (q-1)[\lambda \Gamma(\alpha+1)]^{q-2} \\
& \times \sum_{s=0}^{t-1} \mid f(s+\alpha-1, v(s+\alpha-1)) \\
& \quad-f(s+\alpha-1, u(s+\alpha-1)) \mid \\
\leq & (q-1)[\lambda \Gamma(\alpha+1)]^{q-2} \sum_{s=0}^{t-1} k\|v-u\| \\
\leq & k(q-1)(b+1)[\lambda \Gamma(\alpha+1)]^{q-2}\|v-u\| .
\end{aligned}
$$

Next, for any $u, v \in \mathbb{E}$ and for each $t \in[\alpha-2, \alpha+b+1]_{\mathbb{N}_{\alpha-2}}$, together with the fact that $A(v, u)(0)=0$, we obtain

$$
\begin{aligned}
& |(\mathscr{F} v)(t)-(\mathscr{F} u)(t)| \\
& =\mid \frac{a(t)}{\Gamma(\alpha-1)} \sum_{s=0}^{b+1}(\alpha+b-s-1)^{\frac{\alpha-2}{} A(v, u)(s)} \\
& \quad+\frac{\beta_{1}}{\left(1-\beta_{1}\right) \Gamma(\alpha)} \sum_{s=0}^{b+1}(\alpha+b-s)^{\frac{\alpha-1}{2}} A(v, u)(s) \\
& \quad+\frac{1}{\Gamma(\alpha)} \sum_{s=0}^{t-\alpha}(t-s-1)^{\frac{\alpha-1}{}} A(v, u)(s) \mid
\end{aligned}
$$

$$
\begin{aligned}
& =\mid \frac{a(t)}{\Gamma(\alpha-1)} \sum_{s=1}^{b+1}(\alpha+b-s-1)^{\frac{\alpha-2}{2}} A(v, u)(s) \\
& +\frac{\beta_{1}}{\left(1-\beta_{1}\right) \Gamma(\alpha)} \sum_{s=1}^{b+1}(\alpha+b-s)^{\frac{\alpha-1}{}} A(v, u)(s) \\
& +\frac{1}{\Gamma(\alpha)} \sum_{s=1}^{t-\alpha}(t-s-1)^{\frac{\alpha-1}{\alpha}} A(v, u)(s) \mid \\
& \leq\left\{\frac{|a(t)|}{\Gamma(\alpha-1)} \sum_{s=1}^{b+1}(\alpha+b-s-1)^{\frac{\alpha-2}{2}}\right. \\
& +\frac{\left|\beta_{1}\right|}{\left|1-\beta_{1}\right| \Gamma(\alpha)} \sum_{s=1}^{b+1}(\alpha+b-s)^{\frac{\alpha-1}{}} \\
& \left.+\frac{1}{\Gamma(\alpha)} \sum_{s=1}^{t-\alpha}(t-s-1)^{\frac{\alpha-1}{}}\right\} \\
& \times k(q-1)(b+1)[\lambda \Gamma(\alpha+1)]^{q-2}\|v-u\| \\
& \leq\left\{\frac{\bar{a}(\alpha+b-1)^{\frac{\alpha-1}{u}}}{\Gamma(\alpha)}\right. \\
& \left.+\frac{\left|\beta_{1}\right|(\alpha+b)^{\underline{\alpha}}}{\left|1-\beta_{1}\right| \Gamma(\alpha+1)}+\frac{(\alpha+b)^{\underline{\alpha}}}{\Gamma(\alpha+1)}\right\} \\
& \times k(q-1)(b+1)[\lambda \Gamma(\alpha+1)]^{q-2}\|v-u\| \\
& =\frac{\left\{\left|1-\beta_{1}\right|[\bar{a} \alpha+\alpha+b]+\left|\beta_{1}\right|(\alpha+b)\right\} \Gamma(\alpha+b)}{\left|1-\beta_{1}\right| \Gamma(\alpha+1) \Gamma(b+1)} \\
& \times k(q-1)(b+1)[\lambda \Gamma(\alpha+1)]^{q-2}\|v-u\| \\
& =\left(\left(k ( q - 1 ) ( b + 1 ) \left[\left|1-\beta_{1}\right|(\bar{a}+\alpha+b)\right.\right.\right. \\
& \left.+\left|\beta_{1}\right|(\alpha+b)\right][\lambda \Gamma(\alpha+1)]^{q-2} \\
& \left.\left.\times \prod_{i=1}^{b-1}(\alpha+i)\right) \times\left(\left|1-\beta_{1}\right| b !\right)^{-1}\right)\|v-u\| \\
& =L\|v-u\| \text {, }
\end{aligned}
$$

where $L=\left(k(q-1)(b+1)\left[\left|1-\beta_{1}\right|(\bar{a} \alpha+\alpha+b)+\left|\beta_{1}\right|(\alpha+\right.\right.$ b)] $\left.[\lambda \Gamma(\alpha+1)]^{q-2} \prod_{i=1}^{b-1}(\alpha+i)\right) /\left(\left|1-\beta_{1}\right| b\right.$ !). From (20), we get that $0<L<1$, which implies that $\mathscr{F}$ is a contraction mapping. By means of the Banach contraction mapping principle, we get that $\mathscr{F}$ has a unique fixed point in $\mathbb{E}$; that is. the problem (1) has a unique solution. This completes the proof.

With a similar proof to that of Theorem 6 , we can get the following theorem.

Theorem 7. Suppose $p>2, \beta_{1} \neq 1, \beta_{2} \neq 1$, and the following condition holds: 
$\left(\mathrm{H}_{2}\right)$ there exist constants $\lambda>0$ and $k$ with

$$
\begin{aligned}
0< & k< \\
\times( & \left(\left|1-\beta_{1}\right| b !\right) \\
& \times\left[\left|1-\beta_{1}\right|(\bar{a} \alpha+\alpha+b)+\left|\beta_{1}\right|(\alpha+b)\right] \\
& \left.\times[\lambda \Gamma(\alpha+1)]^{q-2} \prod_{i=1}^{b-1}(\alpha+i)\right)^{-1},
\end{aligned}
$$

such that

$$
\begin{aligned}
& f(t, u) \\
& \quad \leq-\lambda \alpha t \frac{\alpha-1}{,}, \quad \text { for }(t, u) \in[\alpha-1, \alpha+b-1]_{\mathbb{N}_{\alpha-1}} \times \mathbb{R}, \\
& |f(t, v)-f(t, u)| \\
& \quad \leq k|v-u|, \quad \text { for } t \in[\alpha-1, b+\alpha-1]_{\mathbb{N}_{\alpha-1}}, u, v \in \mathbb{R} .
\end{aligned}
$$

Then the problem (1) has a unique solution.

Theorem 8. Suppose $1<p<2, \beta_{1} \neq 1, \beta_{2} \neq 1$, and the following condition holds:

$\left(\mathrm{H}_{3}\right)$ there exists a nonnegative function $g:[\alpha-1, \alpha+b-$ $1]_{\mathbb{N}_{\alpha-1}} \rightarrow \mathbb{R}$ and $\sum_{s=0}^{b} g(s+\alpha-1)=M>0$ such that

$$
|f(t, u)| \leq g(t), \quad(t, u) \in[\alpha-1, \alpha+b-1]_{\mathbb{N}_{\alpha-1}} \times \mathbb{R},
$$

and there exists a positive constant $k$ such that

$$
\begin{aligned}
& |f(t, v)-f(t, u)| \\
& \quad \leq k|v-u|, \quad \text { for } t \in[\alpha-1, \alpha+b-1]_{\mathbb{N}_{\alpha-1}}, u, v \in \mathbb{R} .
\end{aligned}
$$

Then the problem (1) has a unique solution provided that

$$
\begin{aligned}
k<\left(\left|1-\beta_{1}\right| b !\right) & \\
\times( & {\left[\left|1-\beta_{1}\right|(\bar{a} \alpha+\alpha+b)+\left|\beta_{1}\right|(\alpha+b)\right] } \\
& \left.\times(q-1)(b+1) M^{q-2} \prod_{i=1}^{b-1}(\alpha+i)\right)^{-1} .
\end{aligned}
$$

Proof. By (28), we can get that, for $t \in[1, b+1]_{\mathbb{N}_{1}}$,

$$
\begin{gathered}
\left|\sum_{s=0}^{t-1} f(s+\alpha-1, u(s+\alpha-1))\right| \\
\quad \leq \sum_{s=0}^{t-1}|f(s+\alpha-1, u(s+\alpha-1))| \\
\leq \sum_{s=0}^{b} g(s+\alpha-1)=M .
\end{gathered}
$$

In view of $1<p<2$ and $1 / p+1 / q=1$, we can get $q>2$. From (15) and (29), for any $v, u \in \mathbb{E}$, we have

$$
\begin{aligned}
|A(v, u)(t)| \leq & (q-1) M^{q-2} \\
& \times \mid \sum_{s=0}^{t-1} f(s+\alpha-1, v(s+\alpha-1)) \\
& \quad-\sum_{s=0}^{t-1} f(s+\alpha-1, u(s+\alpha-1)) \mid \\
\leq & (q-1) M^{q-2} \\
& \times \sum_{s=0}^{t-1} \mid f(s+\alpha-1, v(s+\alpha-1)) \\
\leq & (q-1) M^{q-2} \sum_{s=0}^{t-1} k\|v-u\| \\
\leq & k(q-1) M^{q-2} t\|v-u\|, \quad t \in[1, b+1]_{\mathbb{N}_{1}}
\end{aligned}
$$

Hence, for any $t \in[\alpha-2, \alpha+b+1]_{\mathbb{N}_{\alpha-2}}$, by $A(u, v)(0)=0$, we have

$$
\begin{aligned}
& |(\mathscr{F} v)(t)-(\mathscr{F} u)(t)| \\
& =\mid \frac{a(t)}{\Gamma(\alpha-1)} \sum_{s=1}^{b+1}(\alpha+b-s-1)^{\frac{\alpha-2}{}} A(v, u)(s) \\
& +\frac{\beta_{1}}{\left(1-\beta_{1}\right) \Gamma(\alpha)} \sum_{s=1}^{b+1}(\alpha+b-s)^{\frac{\alpha-1}{}} A(v, u)(s) \\
& +\frac{1}{\Gamma(\alpha)} \sum_{s=1}^{t-\alpha}(t-s-1) \frac{\alpha-1}{} A(v, u)(s) \\
& \leq\left\{\frac{|a(t)|}{\Gamma(\alpha-1)} \sum_{s=1}^{b+1}(\alpha+b-s-1)^{\frac{\alpha-2}{2}}\right. \\
& +\frac{\left|\beta_{1}\right|}{\left(\left|1-\beta_{1}\right|\right) \Gamma(\alpha)} \sum_{s=1}^{b+1}(\alpha+b-s)^{\frac{\alpha-1}{}} \\
& \left.+\frac{1}{\Gamma(\alpha)} \sum_{s=1}^{t-\alpha}(t-s-1)^{\frac{\alpha-1}{}}\right\} \\
& \times k(q-1)(b+1) M^{q-2}\|v-u\| \\
& \leq\left\{\frac{\bar{a}(\alpha+b-1)^{\frac{\alpha-1}{}}}{\Gamma(\alpha)}\right. \\
& \left.+\frac{\left|\beta_{1}\right|(\alpha+b)^{\underline{\alpha}}}{\left(\left|1-\beta_{1}\right|\right) \Gamma(\alpha+1)}+\frac{(\alpha+b)^{\underline{\alpha}}}{\Gamma(\alpha+1)}\right\} \\
& \times k(q-1)(b+1) M^{q-2}\|v-u\|
\end{aligned}
$$




$$
\begin{gathered}
=\left(\left(k\left[\left|1-\beta_{1}\right|(\bar{a} \alpha+\alpha+b)+\left|\beta_{1}\right|(\alpha+b)\right]\right.\right. \\
\left.\times(q-1)(b+1) M^{q-2} \prod_{i=1}^{b-1}(\alpha+i)\right) \\
\left.\times\left(\left|1-\beta_{1}\right| b !\right)^{-1}\right)\|v-u\|=L\|v-u\|,
\end{gathered}
$$

where $L=\left(k\left[\left|1-\beta_{1}\right|(\bar{a} \alpha+\alpha+b)+\left|\beta_{1}\right|(\alpha+b)\right](q-\right.$ $\left.1)(b+1) M^{q-2} \prod_{i=1}^{b-1}(\alpha+i)\right) /\left(\left|1-\beta_{1}\right| b\right.$ !). In view of $(30), \mathscr{F}$ is a contraction. Thus, the conclusion of the theorem follows by the contraction mapping principle. This completes the proof.

\section{Examples}

In this section, we will illustrate the possible application of the above established analytical results with the following two concrete examples.

Example 1. Consider the discrete fractional boundary value problem

$$
\begin{aligned}
\Delta\left[\phi_{3}\left(\Delta_{C}^{3 / 2} u\right)\right](t) & \\
= & 3\left(t+\frac{1}{2}\right)^{\frac{1 / 2}{}} \\
\times & {\left[\frac{1}{2}+\sin ^{2}\left(\frac{u(t+1 / 2)}{10 \sqrt{3}}+\theta\right)\right.} \\
& \left.+\frac{1}{390}\left|u\left(t+\frac{1}{2}\right)\right|\right], \quad t \in[0,2]_{\mathbb{N}_{0}}, \\
10 u\left(-\frac{1}{2}\right)=u\left(\frac{9}{2}\right), & \\
10 \Delta u\left(-\frac{1}{2}\right) & =10 \Delta u\left(\frac{1}{2}\right)=\Delta u\left(\frac{7}{2}\right),
\end{aligned}
$$

here $\theta$ is a real number.

Conclusion. Problem (34) has a unique nonnegative solution.

Proof. Corresponding to problem (1), $p=3>2, q=3 / 2, \alpha=$ $3 / 2, \beta_{1}=1 / 10, \beta_{2}=1 / 10, b=2$, and $f(t, u)=3 t \frac{1 / 2}{2}[1 / 2+$ $\left.\sin ^{2}((u / 10 \sqrt{3})+\theta)+(1 / 390)|u|\right],(t, u) \in[1 / 2,5 / 2]_{\mathbb{N}_{1 / 2}} \times \mathbb{R}$.

Choosing $\lambda=1$ and $k=3 / 100$, by direct calculation, we can verify that

$$
\begin{aligned}
k= & \frac{3}{100}<\left(\left|1-\beta_{1}\right| b !\right) \\
& \times((q-1)(b+1) \\
& \times\left[\left|1-\beta_{1}\right|(\bar{a} \alpha+\alpha+b)+\left|\beta_{1}\right|(\alpha+b)\right] \\
& \left.\times[\lambda \Gamma(\alpha+1)]^{q-2} \prod_{i=1}^{b-1}(\alpha+i)\right)^{-1} \\
= & \frac{18 \sqrt{3} \pi^{1 / 4}}{325} \approx 0.1277 .
\end{aligned}
$$

It is easy to verify that

$$
\begin{aligned}
& \lambda \alpha t^{\alpha-1}=\frac{3}{2 t \underline{1 / 2}} \\
& \leq 3 t \frac{1 / 2}{2}\left[\frac{1}{2}+\sin ^{2}\left(\frac{u}{10 \sqrt{3}}+\theta\right)+\frac{1}{390}|u|\right] \\
& =f(t, u), \quad(t, u) \in\left[\frac{1}{2}, \frac{5}{2}\right]_{\mathbb{N}_{1 / 2}} \times \mathbb{R}, \\
& |f(t, v)-f(t, u)| \\
& =3 t \frac{1 / 2}{\sin ^{2}}\left(\frac{v}{10 \sqrt{3}}+\theta\right) \\
& -\sin ^{2}\left(\frac{u}{10 \sqrt{3}}+\theta\right) \\
& +\frac{1}{390}|v|-\frac{1}{390}|u| \mid \\
& \leq 3\left(\frac{5}{2}\right)^{\frac{1 / 2}{300}}\left(\frac{1}{390}\right)|v-u| \\
& =\frac{69}{4160} \sqrt{\pi}|v-u| \\
& \approx 0.0294|v-u|<k|v-u|,
\end{aligned}
$$

for $t \in[1 / 2,5 / 2]_{\mathbb{N}_{1 / 2}}, u, v \in \mathbb{R}$. Therefore, by Theorem 6 , the boundary value problem (34) has a unique solution. Furthermore, from the nonnegativeness of $f$ and the expression of $\mathscr{F}$, we also get that the unique solution of (34) is nonnegative.

Example 2. Consider the nonlinear discrete fractional boundary value problem

$$
\begin{aligned}
& \Delta\left[\phi_{3 / 2}\left(\Delta_{C}^{3 / 2} u\right)\right](t) \\
& =\frac{3}{2}\left(t+\frac{1}{2}\right)^{\frac{1 / 2}{2}} \\
& \quad \times \sin ^{2}\left(\frac{u(t+1 / 2)}{40}+\omega\right), \quad t \in[0,2]_{\mathbb{N}_{0}}, \\
& \quad 2 u\left(-\frac{1}{2}\right)=-u\left(\frac{9}{2}\right), \\
& 2 \Delta u\left(-\frac{1}{2}\right)=2 \Delta u\left(\frac{1}{2}\right)=\Delta u\left(\frac{7}{2}\right),
\end{aligned}
$$

where $\omega$ is a real number.

\section{Conclusion. Problem (37) has a unique solution.}

Proof. The problem (37) can be regarded as problem (1), where $p=3 / 2<2, q=3>2, \alpha=3 / 2, \beta_{1}=-1 / 2$, $\beta_{2}=1 / 2, b=2$, and $f(t, u)=(3 / 2) t \frac{1 / 2}{2} \sin ^{2}((u / 40)+\omega),(t, u)$ 
$\in[1 / 2,5 / 2]_{\mathbb{N}_{1 / 2}} \times \mathbb{R}$. Taking $g(t)=(3 / 2) t \frac{1 / 2}{2}, t \in[1 / 2,5 / 2]_{\mathbb{N}_{1 / 2}}$, then $M=(105 \Gamma(1 / 2)) / 32$. Let $k=1 / 600 \approx 0.0017$; we have

$$
\begin{aligned}
& k<\left(\left|1-\beta_{1}\right| b !\right) \\
& \times\left(\left[\left|1-\beta_{1}\right|(\bar{a} \alpha+\alpha+b)+\left|\beta_{1}\right|(\alpha+b)\right]\right. \\
& \left.\quad \times(q-1)(b+1) M^{q-2} \prod_{i=1}^{b-1}(\alpha+i)\right)^{-1} \\
& =\frac{64}{15225 \sqrt{\pi}} \approx 0.0024 .
\end{aligned}
$$

Moreover, we can verify that

$$
\begin{aligned}
|f(t, u)| \leq g(t), & (t, u) \in\left[\frac{1}{2}, \frac{5}{2}\right]_{\mathbb{N}_{1 / 2}} \times \mathbb{R}, \\
|f(t, v)-f(t, u)| \leq & \frac{3}{2} t \frac{1 / 2}{40^{2}}|v-u| \\
& \leq \frac{3}{2}\left(\frac{5}{2}\right)^{\frac{1 / 2}{2}} \frac{1}{40^{2}}|v-u| \\
& =\frac{9 \sqrt{\pi}}{10240}|v-u| \\
& \approx 0.0016|v-u|<k|v-u|,
\end{aligned}
$$

for $t \in[1 / 2,5 / 2]_{\mathbb{N}_{1 / 2}}, v, u \in \mathbb{R}$. tion.

Therefore, by Theorem 8, problem (37) has a unique solu-

\section{Acknowledgment}

This work was supported by the Longdong University Grant XYZK-1010 and XYZK-1007.

\section{References}

[1] S. G. Samko, A. A. Kilbas, and O. I. Marichev, Fractional Integrals and Derivatives: Theory and Applications, Gordon and Breach Science, Yverdon, Switzerland, 1993.

[2] W. G. Glockle and T. F. Nonnenmacher, "A fractional calculus approach to self-similar protein dynamics," Biophysical Journal, vol. 68 , no. 1 , pp. 46-53, 1995.

[3] R. Metzler, W. Schick, H. Kilian, and T. F. Nonnenmacher, "Relaxation in filled polymers: a fractional calculus approach," The Journal of Chemical Physics, vol. 103, no. 16, pp. 7180-7186, 1995.

[4] I. Podlubny, Fractional Differential Equations, vol. 198 of Mathematics in Science and Engineering, Academic Press, San Diego, Calif, USA, 1999.

[5] R. Hilfer, Applications of Fractional Calculus in Physics, World Scientific, Singapore, 2000.

[6] A. A. Kilbas and J. J. Trujillo, "Differential equations of fractional order: methods, results and problems. I," Applicable Analysis, vol. 78, no. 1-2, pp. 153-192, 2001.

[7] A. A. Kilbas and J. J. Trujillo, "Differential equations of fractional order: methods, results and problems. II," Applicable Analysis, vol. 81, no. 2, pp. 435-493, 2002.
[8] J. Sabatier, O. P. Agrawal, and J. A. T. Machado, Advances in Fractional Calculus: Theoretical Developments and Applications in Physics and Engineering, Springer, Heidelberg, Germany, 2007.

[9] Z. Bai and H. Lü, "Positive solutions for boundary value problem of nonlinear fractional differential equation," Journal of Mathematical Analysis and Applications, vol. 311, no. 2, pp. 495-505, 2005.

[10] Z. Bai, "On positive solutions of a nonlocal fractional boundary value problem," Nonlinear Analysis: Theory, Methods \& Applications A, vol. 72, no. 2, pp. 916-924, 2010.

[11] Z. Bai and Y. Zhang, "Solvability of fractional three-point boundary value problems with nonlinear growth," Applied Mathematics and Computation, vol. 218, no. 5, pp. 1719-1725, 2011.

[12] C. Bai, "Triple positive solutions for a boundary value problem of nonlinear fractional differential equation," Electronic Journal of Qualitative Theory of Differential Equations, vol. 2008, article 24,2008

[13] X. Xu, D. Jiang, and C. Yuan, "Multiple positive solutions for the boundary value problem of a nonlinear fractional differential equation," Nonlinear Analysis: Theory, Methods \& Applications $A$, vol. 71, no. 10, pp. 4676-4688, 2009.

[14] B. Ahmad, "Existence of solutions for irregular boundary value problems of nonlinear fractional differential equations," Applied Mathematics Letters of Rapid Publication, vol. 23, no. 4, pp. 390394, 2010.

[15] S. Zhang, "Positive solutions to singular boundary value problem for nonlinear fractional differential equation," Computers \& Mathematics with Applications, vol. 59, no. 3, pp. 1300-1309, 2010.

[16] W. Jiang, "The existence of solutions to boundary value problems of fractional differential equations at resonance," Nonlinear Analysis: Theory, Methods \& Applications A, vol. 74, no. 5, pp. 1987-1994, 2011.

[17] Y. Chen and X. Tang, "Solvability of sequential fractional order multi-point boundary value problems at resonance," Applied Mathematics and Computation, vol. 218, no. 14, pp. 7638-7648, 2012.

[18] A. Guezane-Lakoud and R. Khaldi, "Solvability of a fractional boundary value problem with fractional integral condition," Nonlinear Analysis: Theory, Methods \& Applications A, vol. 75, no. 4, pp. 2692-2700, 2012.

[19] R. Hilscher and V. Zeidan, "Nonnegativity and positivity of quadratic functionals in discrete calculus of variations: survey," Journal of Difference Equations and Applications, vol. 11, no. 9, pp. 857-875, 2005.

[20] W. G. Kelley and A. C. Peterson, Difference Equations: An Introduction with Applications, Academic Press, New York, NY, USA, 1991.

[21] F. M. Atici and P. W. Eloe, "A transform method in discrete fractional calculus," International Journal of Difference Equations, vol. 2, no. 2, pp. 165-176, 2007.

[22] F. M. Atici and P. W. Eloe, "Initial value problems in discrete fractional calculus," Proceedings of the American Mathematical Society, vol. 137, no. 3, pp. 981-989, 2009.

[23] F. M. Atıcı and P. W. Eloe, "Discrete fractional calculus with the nabla operator," Electronic Journal of Qualitative Theory of Differential Equations, vol. 2009, article 3, 12 pages, 2009.

[24] F. M. Atıcı and S. Şengül, "Modeling with fractional difference equations," Journal of Mathematical Analysis and Applications, vol. 369, no. 1, pp. 1-9, 2010. 
[25] F. M. Atıcı and P. W. Eloe, "Two-point boundary value problems for finite fractional difference equations," Journal of Difference Equations and Applications, vol. 17, no. 4, pp. 445-456, 2011.

[26] C. S. Goodrich, "Solutions to a discrete right-focal fractional boundary value problem," International Journal of Difference Equations, vol. 5, no. 2, pp. 195-216, 2010.

[27] C. S. Goodrich, "Continuity of solutions to discrete fractional initial value problems," Computers \& Mathematics with Applications, vol. 59, no. 11, pp. 3489-3499, 2010.

[28] C. S. Goodrich, "Existence and uniqueness of solutions to a fractional difference equation with nonlocal conditions," Computers \& Mathematics with Applications, vol. 61, no. 2, pp. 191-202, 2011.

[29] C. S. Goodrich, "Existence of a positive solution to a system of discrete fractional boundary value problems," Applied Mathematics and Computation, vol. 217, no. 9, pp. 4740-4753, 2011.

[30] C. S. Goodrich, "On a discrete fractional three-point boundary value problem," Journal of Difference Equations and Applications, vol. 18, no. 3, pp. 397-415, 2012.

[31] C. S. Goodrich, "On discrete sequential fractional boundary value problems," Journal of Mathematical Analysis and Applications, vol. 385, no. 1, pp. 111-124, 2012.

[32] F. Chen, X. Luo, and Y. Zhou, "Existence results for nonlinear fractional difference equation," Advances in Difference Equations, vol. 2011, Article ID 713201, 12 pages, 2011.

[33] N. R. O. Bastos, R. A. C. Ferreira, and D. F. M. Torres, "Discretetime fractional variational problems," Signal Process, vol. 91, no. 3, pp. 513-524, 2011.

[34] N. R. O. Bastos, R. A. C. Ferreira, and D. F. M. Torres, "Necessary optimality conditions for fractional difference problems of the calculus of variations," Discrete and Continuous Dynamical Systems, vol. 29, no. 2, pp. 417-437, 2011.

[35] T. Abdeljawad, "On Riemann and Caputo fractional differences," Computers \& Mathematics with Applications, vol. 62, no. 3, pp. 1602-1611, 2011.

[36] M. Holm, "Sum and difference compositions in discrete fractional calculus," Cubo, vol. 13, no. 3, pp. 153-184, 2011.

[37] R. A. C. Ferreira, "Existence and uniqueness of solution to some discrete fractional boundary value problems of order less than one," Journal of Difference Equations and Applications, vol. 19, no. 5, pp. 712-718, 2013.

[38] J. Wang, H. Xiang, and Z. Liu, "Existence of concave positive solutions for boundary value problem of nonlinear fractional differential equation with $p$-Laplacian operator," International Journal of Mathematics and Mathematical Sciences, vol. 2010, Article ID 495138, 17 pages, 2010.

[39] J. Wang and H. Xiang, "Upper and lower solutions method for a class of singular fractional boundary value problems with $p$ Laplacian operator," Abstract and Applied Analysis, vol. 2010, Article ID 971824, 12 pages, 2010.

[40] Z. Han, H. Lu, S. Sun, and D. Yang, "Positive solutions to boundary-value problems of $p$-Laplacian fractional differential equations with a parameter in the boundary," Electronic Journal of Differential Equations, vol. 2012, article 213, 14 pages, 2012.

[41] G. Chai, "Positive solutions for boundary value problem of fractional differential equation with $p$-Laplacian operator," Boundary Value Problems, vol. 2012, article 18, 2012.

[42] T. Chen and W. Liu, "An anti-periodic boundary value problem for the fractional differential equation with a $p$-Laplacian operator," Applied Mathematics Letters of Rapid Publication, vol. 25, no. 11, pp. 1671-1675, 2012.
[43] X. Liu and M. Jia, "On the solvability of a fractional differential equation model involving the $p$-Laplacian operator," Computers \& Mathematics with Applications, vol. 64, no. 10, pp. 3267-3275, 2012.

[44] H. Lu, Z. Han, S. Sun, and J. Liu, "Existence on positive solutions for boundary value problems of nonlinear fractional differential equations with $p$-Laplacian," Advances in Difference Equations, vol. 2013, article 30, 2013.

[45] W. Lv, "Existence of solutions for discrete fractional boundary value problems with a $p$-Laplacian operator," Advances in Difference Equations, vol. 2012, article 163, 2012.

[46] K. S. Miller and B. Ross, "Fractional difference calculus," in Proceedings of the International Symposium on Univalent Functions, Fractional Calculus and Their Applications, Ellis Horwood Series in Mathematics \& Its Applications, pp. 139-152, Nihon University, Koriyama, Japan, May 1988, Horwood, Chichester, UK, 1989. 


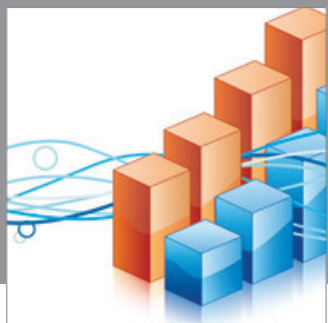

Advances in

Operations Research

mansans

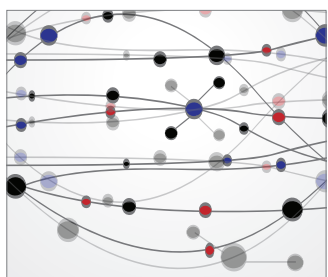

The Scientific World Journal
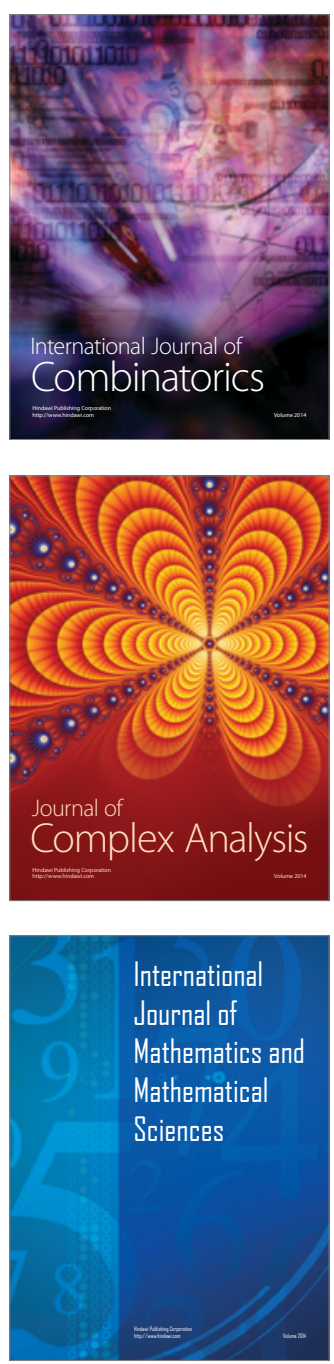
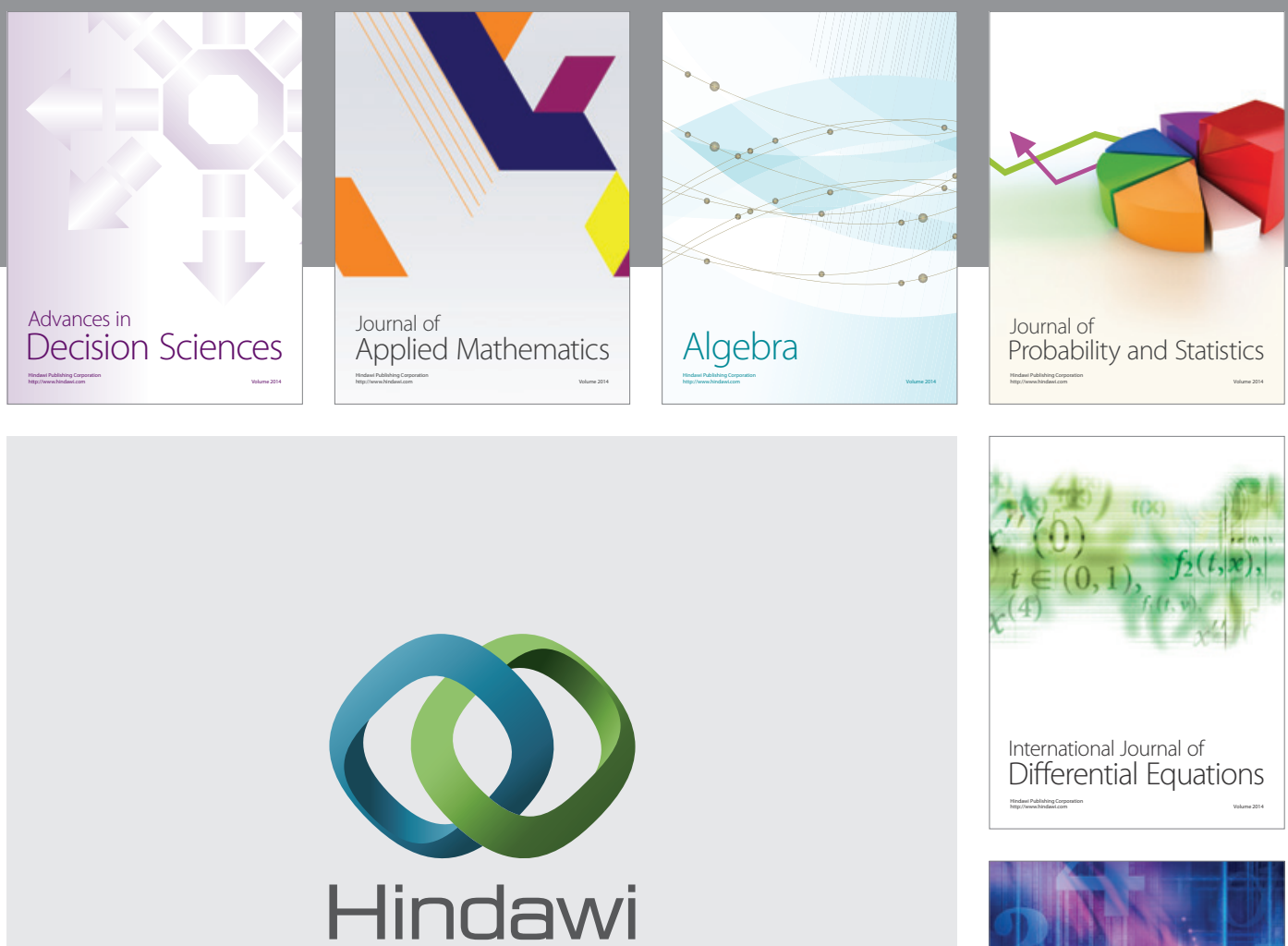

Submit your manuscripts at http://www.hindawi.com
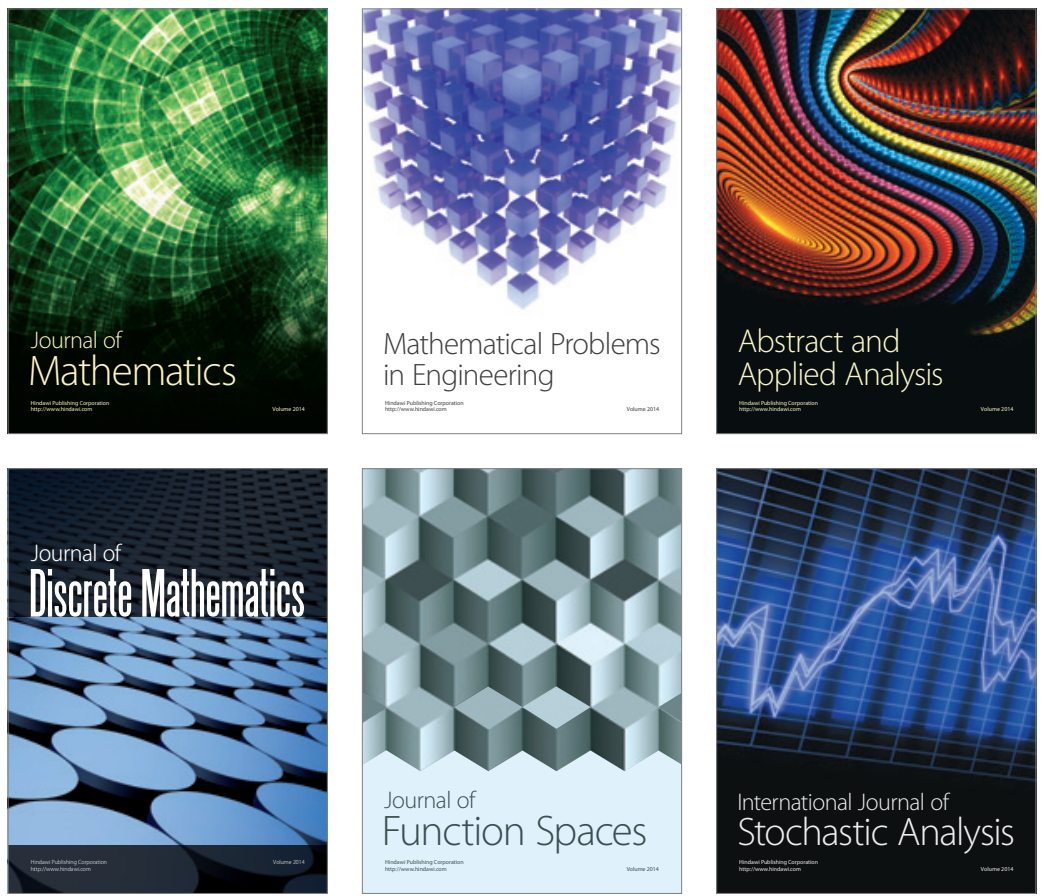

Journal of

Function Spaces

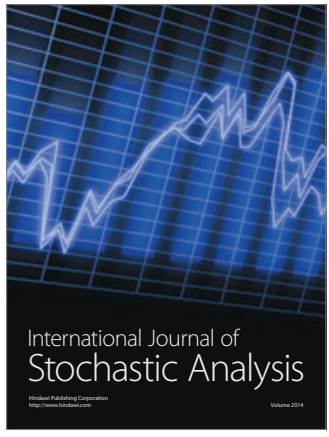

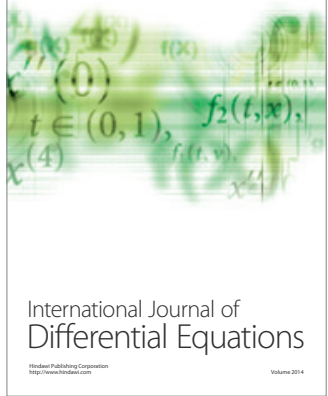
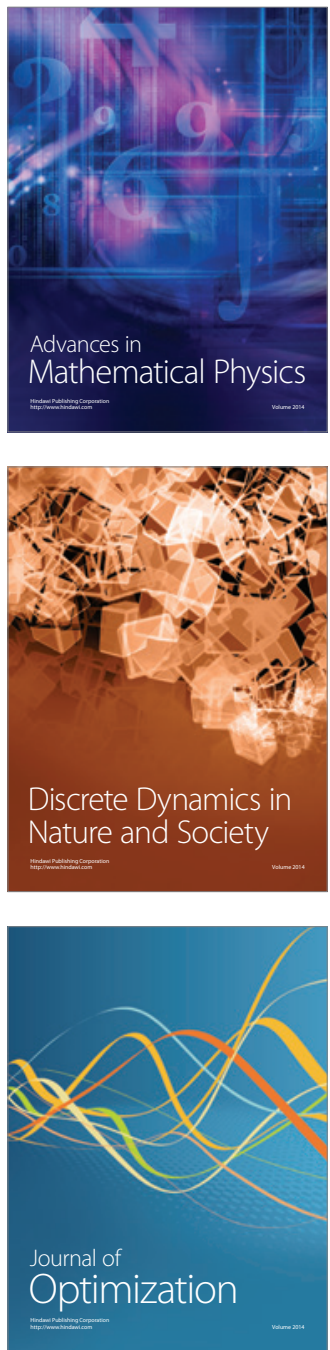